\title{
EL CAMBIO CLIMÁTICO Y LA GANADERÍA BOVINA: UNA EVALUACIÓN PARA EL SUDESTE BONAERENSE, ARGENTINA
}

\section{CLIMATE CHANGE AND CATTLE PRODUCTION: AN EVALUATION FOR SOUTHEASTERN BUENOS AIRES, ARGENTINA}

\author{
Hernán A. Urcola ${ }^{1 *}$, Julio C. Burges ${ }^{1,2}$, Lucie Gouarderes ${ }^{3}$, y Silvina Solman ${ }^{4}$
}

${ }^{1}$ Instituto Nacional de Tecnología Agropecuaria, Ruta 226 km 73,5 (7620), Balcarce, Argentina.

2 Facultad de Ciencias Agrarias, Universidad Nacional de Mar del Plata, Ruta 226 km 73,5 (7620), Balcarce, Argentina.

${ }^{3}$ Universidad SUPAGRO, 2 Place Pierre Viala, 34060, Montpellier, Francia.

${ }^{4}$ Universidad de Buenos Aires, Facultad de Ciencias Exactas y Naturales, Departamento de Ciencias de la Atmósfera y los Océanos DCAO-FCEN-UBA, Buenos Aires, Argentina; CONICET- Universidad de Buenos Aires, Centro de Investigaciones del Mar y la Atmósfera (CIMA/CONICET-UBA), Buenos Aires, Argentina; Instituto Franco Argentino de Estudios del Clima y sus Impactos (UMI IFAECI/ CNRS), Ciudad Universitaria, pabellón II, piso 2, C1428EGA, Buenos Aires, Argentina.

* Autor para correspondencia Email: urcola.hernan@inta.gob.ar

\section{RESUMEN}

No se cuenta con estimaciones de los efectos del cambio climático sobre la producción y el rendimiento económico de la ganadería del sudeste bonaerense. El objetivo de este trabajo fue estimar los efectos del cambio climático sobre la producción y la economía de un predio ganadero de ciclo completo típico de la región. Dada la naturaleza de la investigación, se utilizaron técnicas de modelación a partir de datos climáticos de alta resolución y parámetros productivos para un rebaño de producción del partido (departamento) de Balcarce, Provincia de Buenos Aires, Argentina. Se caracterizó el clima esperado en tres períodos de años, denominados, Histórico, Futuro Cercano y Futuro Lejano, y se modelizó la producción de pasto, de carne y el margen bruto por hectárea. Los resultados indican que la región experimentará un incremento de temperatura mínima (máxima) promedio de $2,6^{\circ} \mathrm{C}\left(1,9^{\circ} \mathrm{C}\right)$ y un incremento del $27 \%$ en las precipitaciones promedio anuales, hacia el año 2100. La producción de pasto, de carne y el margen bruto experimentarían cambios leves, tendiendo a incrementarse hacia finales del siglo. El clima de los períodos futuros analizados no implica un mayor riesgo para la producción ganadera.

Palabras clave: Cambio climático, rebaño de cría, impacto productivo y económico, depresión del Salado.

\section{ABSTRACT}

There are no precise estimates available of the productive and economic impact of climate change effects on the cattle production in the Buenos Aires province. The objective of this study was to estimate the impact of climate change on the productive and economic performance of a representative cattle herd of Southeastern Buenos Aires. Given the nature of this research, a modeling approach using high resolution climatic data and reproductive parameters for a whole-cycle herd was used. The study site was located near the city of Balcarce. Climatic conditions for three periods of years were 
characterized and named as Historical, Near Future and Distant Future. Pasture production, meat production and gross margin were modeled. The results indicate that the region will experience an increase in minimum (maximum) average temperature of $2.6^{\circ} \mathrm{C}\left(1.9^{\circ} \mathrm{C}\right)$, and an increase of $27 \%$ in mean annual rainfall at the end of the twenty first century. Pasture production, meat production and gross margins would experience small changes and even tend to increase by the end of the century. Climatic conditions in the future periods analyzed do not pose a higher production risk when compared to the Historical period.

Key words: Climate change, cow-calf herd, productive and economic impact, Salado depression.

\section{INTRODUCCIÓN}

En la Cuenca del Río Salado, Argentina, la producción ganadera está fuertemente influenciada por las condiciones climáticas. Esta región, de unas 9 millones de hectáreas, caracterizada por limitaciones edáficas en gran parte de su superficie, aporta una importante cantidad de los terneros para engorde de Argentina. En la gran mayoría de los establecimientos, la alimentación animal se basa casi exclusivamente en pasturas y pastizales en condiciones de secano. Por lo tanto, la variabilidad climática, intra e interanual, modifica el crecimiento vegetal afectando la productividad física y económica de los sistemas de producción de la región (Romera et al., 2005a; Burges y Romera, 2007).

En base a las simulaciones del proyecto Intercomparación de Modelos Climáticos Acoplados, Fase 5 (CMIP5, por sus siglas en inglés), el rango del cambio proyectado de temperatura para la región está entre 1 y $4^{\circ} \mathrm{C}$ para finales del siglo XXI, dependiendo del escenario, siendo los cambios proyectados para los meses de verano superiores a los de invierno. Los cambios proyectados por los modelos climáticos regionales forzados por modelos globales pertenecientes al proyecto de Intercomparación de Modelos Acoplados, Fase 3 (CMIP3, por sus siglas en inglés) coinciden en el rango de los cambios de temperatura proyectados para fines de siglo XXI (Sánchez et al., 2015).

Tanto las proyecciones provistas por modelos globales como por modelos regionales coinciden en proyectar un incremento de precipitación de entre el 10 y el $40 \%$ en el sudeste de Sudamérica durante los meses de verano. Para los meses de invierno la mayoría de los modelos proyectan incrementos de entre 10 y $20 \%$ en un área menor, en comparación con el verano (Christensen et al., 2013; Sánchez et al., 2015). El acuerdo en las proyecciones de precipitación entre diferentes grupos de modelos, tanto globales como regionales en la región del sudeste de Sudamérica permite asignar un alto nivel de confiabilidad a los cambios proyectados. Además de los cambios en precipitación y temperatura mencionados para Sudamérica, se proyecta también un incremento en la frecuencia de noches cálidas y un incremento en los eventos de precipitación extrema (Re and Barros, 2009; Marengo et al., 2009; Skansi et al., 2013)

Para la Región Pampeana de Argentina, la evidencia existente sobre los posibles efectos del cambio climático en la ganadería presenta conclusiones contradictorias. Por un lado, Giménez (2006), basándose en proyecciones de modelos climáticos globales y en el escenario A2 del Reporte Especial sobre Escenarios de Emisiones (SRES, por sus siglas en inglés), estima que la producción de pastos y pastizales podría incrementarse entre 1\% y 9\%, para el año 2020. Sin embargo, Seo (2009) y Seo et al. (2010) indican que tanto el stock bovino argentino como su valor podrían reducirse bajo determinados escenarios climáticos. Esta reducción ocurriría porque sería óptimo para los productores sustituir hectáreas ganaderas con hectáreas de cultivos. Más allá de estas referencias, no se ha estimado el impacto esperado con proyecciones de alta resolución con modelos regionales de última generación. Tampoco se han considerado las interacciones entre clima, pastura y animales de manera sistémica, ni cómo las capacidades de amortiguación y regulación de estos componentes se combinan para determinar el rendimiento físico y económico del rebaño.

De acuerdo a los antecedentes descritos, el objetivo de este trabajo es estimar el impacto productivo y económico del cambio climático sobre un sistema de producción ganadera de ciclo completo del sudeste bonaerense. La evaluación de impacto del cambio climático permitirá diseñar medidas de adaptación que limiten posibles efectos negativos sobre la producción.

\section{MATERIALES Y MÉTODOS}

Dada la naturaleza del trabajo, se utilizaron técnicas de simulación para responder al objetivo planteado.

\section{Clima}

El clima se caracterizó a través las simulaciones climáticas realizadas para SudAmerica utilizando el modelo climático regional 
Rossby Centre Regional Climate model versión 3.5 (de aquí en adelante, RCA3) (Samuelsson et al., 2011), desarrollado por el Instituto Meteorológico e Hidrológico de Suecia (SMHI, por sus sigla en inglés). El RCA3 es un modelo climático regional que simula los principales procesos físicos que determinan el clima de una región dada, durante un lapso de tiempo. (Una explicación resumida del funcionamiento de los modelos climáticos globales y regionales puede encontrarse en: $\mathrm{http}: / /$ www.fing.edu.uy/cluster/eolica/stars/index5. html) El RCA3 se basa en una estructura de mosaicos cada uno de los cuales se corresponde con un prisma que abarca desde una profundidad de 3 metros por debajo de la superficie del suelo hasta altos niveles de la atmósfera. Cada uno de estos mosaicos representan distintos tipos de superficie y de suelo (océano, hielo, bosques o tierras con distinta vegetación) y el modelo resuelve para cada uno de ellos las ecuaciones de balance mecánico, balance térmico, y balance de especies relevantes a la atmósfera, como la humedad o la concentración de sustancias químicas o aerosoles en la atmósfera. Cada uno de estos balances se integra en forma dinámica para predecir las variables que describen el clima de la región. El RCA3 funciona de forma óptima con resoluciones de $10 \mathrm{a} 50 \mathrm{~km}$ y con un paso de 30 minutos (Jones et al., 2004; Samuelsson et al. 2011).

El modelo RCA3 ha sido evaluado y puesto a punto en el marco del Proyecto CLARIS LPB (A Europe-South America Network for Climate Change Assessment and Impact studies in $\mathrm{La}$ Plata Basin, http://eolo.cima.fcen.uba.ar/ sweb/) (Solman et al., 2013). Las proyecciones del clima de Argentina generadas por el modelo RCA3 son comparables en términos de los cambios proyectados de temperatura y precipitación medias con las de otros modelos climáticos regionales (Sánchez et al., 2015). Se utilizaron las salidas de la simulación climática provista por el modelo RCA3 anidada en el modelo global EC5OM para el escenario futuro de emisión SRESA1B para el periodo 1961-2100, realizada en el marco del Proyecto CLARIS-LPB. Para el presente análisis se seleccionó el punto $-37.9858^{\circ} \mathrm{S}$ y $-58.5736^{\circ} \mathrm{O}$, ubicado a unos $32 \mathrm{~km}$ al sudoeste de la ciudad de Balcarce. El clima del sitio se caracterizó a través de las variables: temperatura mínima, temperatura máxima y precipitación, ya que estas variables son las que explican la mayor parte de la variabilidad en el rendimiento de cultivos y pasturas (Magrin et al., 2005). Se caracterizó y comparó el régimen térmico y pluviométrico de tres períodos de años, denominados: Histórico, 1971-2000; Futuro Cercano, 2011-2040; y Futuro Lejano, 2071-2100.
La comparación de períodos de 30 años ayuda a evaluar los efectos de la variabilidad interanual y los efectos de la variabilidad inter-decadal en los procesos climáticos (CLARIS-LPB, 2010).

Debido a que el modelo RCA3 presenta errores sistemáticos en la simulación del clima presente (Solman et al., 2013; Sánchez et al., 2015), se aplicó la corrección denominada delta-change (Hay et al., 2000) tanto para las condiciones del clima presente como para el clima futuro. Este tipo de correcciones consiste en aplicar los cambios generados por los modelos climáticos a las series históricas de cada variable y así obtener las series de tiempo futuras de estas variables. Numerosos estudios indican que las evaluaciones de impacto, como las realizadas aquí, son más precisas utilizando correcciones del tipo deltachange (Michelangeli et al., 2009; Yang et al., 2010; Oettli et al., 2011).

Para evaluar la robustez de la señal del cambio climático, considerando que se utilizan los cambios proyectados basados en un único modelo climático regional, se computó el cociente entre el cambio proyectado y la variabilidad interanual:

$$
R_{\text {var }}=\left.\left|\Delta \overline{\operatorname{Var}}_{\text {Fut-Press }}\right|\right|_{S T D V_{\text {var }},},
$$

dónde $\Delta \overline{\operatorname{Var}}_{\text {Fut-Press }}$ es la diferencia entre el clima en el escenario futuro y el clima en el presente y $S T D V_{\text {var }}$ es la desviación estándar de la proyección climática (Hayashi, 1982; Cabré et al., 2016). De esta forma, valores de $\boldsymbol{R}_{\text {var }}$ mayores a 1 indican que la señal de cambio climático es robusta, ya que su magnitud de cambio es mayor a la variabilidad interanual. Por el contrario, valores menores a 1 indican señales de cambio poco robustas asociadas con procesos climáticos de mayor incertidumbre.

\section{Producción de pasto}

Para cada uno de los períodos considerados, se simuló el crecimiento de una pastura de agropiro (Thinopirum ponticum, Podp.) utilizando el modelo propuesto por Romera et al. (2009). Este modelo considera la interacción de variables climáticas con parámetros del suelo y de la especie forrajera simulada, permitiendo así ajustar el modelo a un sitio determinado. Estos parámetros reflejan la influencia de la fertilidad del suelo, la composición de las especies y su patrón estacional de crecimiento. De esta forma, la pastura es caracterizada como un sistema dinámico conformado por tejido vivo y muerto. El tejido vivo ingresa al sistema como resultado de la fotosíntesis, y si ese tejido no es cosechado, por pastoreo o corte, entra en senescencia e ingresa al 
pool de tejido muerto. El tejido muerto se pierde del sistema vía fauna del suelo, descomposición y lixiviación.

El modelo utilizado tiene como variable de estado la masa de pastura viva y cuya tasa de cambio neto es determinada por el balance entre la incorporación de nuevo crecimiento de pasto y la tasa diaria de senescencia. El modelo estima la tasa de senescencia en función de la temperatura y de la vida media foliar de la especie forrajera (Romera et al., 2009) y el total de material que senesce depende de la masa de material vivo en la pastura. El nuevo crecimiento de la pastura (tejido verde o vivo) es función de la temperatura, del índice de área foliar y del balance de agua en el suelo. El índice de área foliar se relaciona con la capacidad de captar radiación solar y se consideró función de la masa de material vivo en la pastura. Para el cálculo del balance hídrico, se consideró una capacidad de campo de $45 \mathrm{~mm}$, diariamente se incorporó el agua de precipitación (cuando fue mayor a $1 \mathrm{~mm}$ ) y se restó la evapotranspiración de agua disponible en el suelo mediante la ecuación de Priestley y Taylor (1972) ya que es recomendada cuando no se dispone de la información climática completa (Alexandris et al., 2008). No se consideraron los procesos de drenaje a capas inferiores, ni la capilaridad, ni el escurrimiento a zonas más bajas.

Este modelo de crecimiento de pasto, programando en Visual Basic ${ }^{\circledR}$, ha sido calibrado para un campo de Balcarce (Reserva 6) y ha sido utilizado para estudiar diferentes estrategias de manejo ganadero en virtud de que logra una representación aceptable del crecimiento vegetal al compararlo con muestras obtenidas en experimentos de corte de pasturas de agropiro (Romera, 2004; Romera et al., 2004; 2008). Este modelo ha permitido analizar diferentes estrategias en sistemas de cría vacuna en los que el manejo forrajero y el clima interactúan (Romera et al., 2005b; 2008).

La producción de pasto se expresó en equivalente vaca $(E V)$, una unidad que expresa la energía metabolizable (EM) del forraje y que es frecuentemente usada por los profesionales en Argentina para la estimación de requerimientos energéticos de los rebaños. Un EV equivale a 18,5 Mcal EM día $^{-1}$. Se consideró un contenido de energía bruta de la dieta de 4,4 en Mcal kg MS $\mathrm{ha}^{-1}$, un factor de pérdidas de energía en orina y metano de 0,82 y un patrón de digestibilidad estacional para pasturas de agropiro de la zona (Romera, 2004).

\section{Producción de carne y margen bruto}

La producción de carne se basó en un rebaño de producción de ciclo completo (es decir, los terneros producidos se engordan) con un manejo y una eficiencia típica de los establecimientos ganaderos de la región (Romera et al., 2008; Tosi, 2017). En este rebaño el servicio de monta se realiza en primavera y el destete a principios de marzo. Se modelizó este rebaño con la posibilidad de variar su composición, y por ende sus requerimientos, ajustando la venta de más o menos terneros al momento del destete. Esta variación en las ventas constituye una estrategia para adaptar los requerimientos del rodeo ante las variaciones del crecimiento de la pastura.

Se consideró un pastoreo rotativo con una asignación forrajera contemplando un aprovechamiento por parte de los animales de $60 \%$ del forraje disponible para considerar pérdidas por pisoteo y desperdicio. Para ajustar la carga animal se utilizó el método del balance forrajero, estimando la receptividad ganadera (RG). La RG es la densidad máxima de animales que pueden vivir en un hábitat determinado (Golluscio, 2009). Se eligió este procedimiento ya que constituye un método muy difundido y de aplicación frecuente entre productores y asesores de la región pampeana. Sin embargo, el cálculo de la RG puede verse afectado por que existe una interacción entre la carga animal asignada a un recurso forrajero y su receptividad. Así, tanto el sobrepastoreo como el subpastoreo pueden llevar a la disminución de la receptividad del recurso, tanto en el período actual como en los subsiguientes (Golluscio, 2009). Para minimizar estas limitaciones, se incorporaron en el modelo reglas de manejo, como proponen Burges et al. (2014), para mantener en todo momento un remanente de forraje disponible en $1000 \mathrm{~kg}$ MS $\mathrm{ha}^{-1}$ para evitar sobrepastoreos. En caso de déficits de forraje, se utilizan reservas forrajeras del año anterior y en caso de agotarse estas, se compran reservas forrajeras en forma de rollos de heno. Además, el modelo incluye un corte para rollos de todo excedente no consumido en los meses de octubre, noviembre y diciembre, para evitar el deterioro de la pastura por encañamiento y exceso de senescencia.

Con las precauciones descriptas, la estimación de la RG a través del balance forrajero constituye un método simple y robusto que conlleva las siguientes ventajas: (i) al calcularse la demanda y la oferta de alimento en términos de energía metabólica toma en cuenta explícitamente la calidad nutritiva de los recursos forrajeros, y (ii) al tratarse de un procedimiento iterativo permite adecuar la cantidad de animales asignados a las raciones existentes. Con esta última característica el método de la RG se vuelve especialmente útil para la toma de decisiones en una escala intraanual, y se adecua perfectamente al diseño de 
nuestro rodeo con proporción variable de recría.

La carga animal se fija en $1,7 \mathrm{EV} \mathrm{ha}^{-1}$ para un rodeo de ciclo completo con $100 \%$ de recría de los machos y hembras de destete. Este valor de carga animal es representativo de los rebaños de ciclo completo típicos de la región bajo estudio (Tosi, 2017). Luego, esta carga animal se ajusta anualmente, variando el número de animales que se venden al destete, de acuerdo a la regla de manejo que considera la disponibilidad de forraje del año anterior (Tabla 1). Es decir, de acuerdo a la disponibilidad de alimento se venden más o menos animales al momento del destete. De esta forma, con $0 \%$ de recría se venden los terneros de ambos sexos al destete; con 50\% de recría se venden todos los terneros de destete engordándose todas las terneras de destete; con $75 \%$ de recría se venden la mitad de los terneros al destete engordándose las terneras y la mitad de los terneros; y con $100 \%$ de recría no se realizan ventas al destete y todos los terneros y las terneras pasan a engordarse en el establecimiento.

La decisión de la cantidad y sexo de animales a vender se toma en los días previos al destete, y los valores límites para determinar la composición del rebaño corresponden a una reserva de aproximadamente el $25 \%$ del forraje extra al requerido por cada uno de los rebaños durante el período invernal (mayo-julio). Este mecanismo de ajuste del número de animales que se engordan es la forma de operación de la mayoría de los establecimientos ganaderos y la carga inicial representa un valor usual para establecimientos ganaderos de la zona (Tosi, 2017).

Los requerimientos energéticos mensuales del rodeo descrito según cada porcentaje de recría se expresaron en EV y se calcularon utilizando los requerimientos nutricionales de los animales considerando su categoría, estado fisiológico y ritmo de ganancia o pérdida de peso de los animales (ARC, 1980; Keaokliang et al. 2018).

Cuando las ventas se realizan al destete los pesos de venta fueron de 160 y $170 \mathrm{~kg} \mathrm{cab}^{-1}$ para hembras y machos, respectivamente; mientras que en el caso de recriarse, hembras y machos se engordaron hasta un peso de 325 y $418 \mathrm{~kg} \mathrm{cab}^{-1}$, respectivamente. Las vacas de descarte se venden al alcanzar los $400 \mathrm{~kg} \mathrm{cab}^{-1}$. En los rodeos de recría y ciclo completo las categorías en engorde reciben suplementación con el $1 \%$ de su peso vivo con maíz durante los últimos 30 días de engorde.

De acuerdo a la carga animal de cada año y la composición del rodeo, se calcula la producción de carne como la diferencia entre las ventas y las compras de animales. La diferencia de inventario se fija en cero en este trabajo, ya que se considera un rodeo estable. Por último, el margen bruto anual del sistema se estima como la diferencia entre el ingreso bruto (peso de los animales vendidos por categoría multiplicado por el precio correspondiente a cada categoría) y los costos de sanidad, confección distribución y compra de rollos, suplementación con maíz, mano de obra, mantenimiento y renovación de la pastura y gastos de comercialización y fletes. Se consideró el calendario de vacunación y las revisaciones preventivas recomendadas por el INTA para rodeos de la región (Quiroz y Rodríguez, 2012). Por simplificación, no se incluyen costos financieros, dado que no se evalúan manejos alternativos que requieran distinto niveles de inversión y su inclusión afectaría a cada período analizado por igual. El margen bruto se expresa en pesos por hectárea.

Para valorar las ventas se utilizan los precios de cada categoría correspondiente a cada mes de cada año de venta correspondiente al período histórico, tomados de la revista Márgenes Agropecuarios (www.margenes.com). Esta misma serie de precios se repite para cada año

Tabla 1. Regla de decisión para determinar la composición del rebaño al momento de destete.

Table 1. Decision rule to determine the herd composition at weaning.

\begin{tabular}{ll}
\hline Condición & Acción \\
\hline Si $[$ EV disponibles en febrero] $+<=450$, & Recría: $0 \% \%^{\ddagger}$ \\
Si $450<[$ EV disponibles en febrero] $<=700$, & Recría: $50 \%$ \\
Si $700<[$ EV disponibles en febrero] $<=900$, & Recría: $75 \%$ \\
Si $900<[$ EV disponibles en febrero], & Recría: $100 \%$ \\
\hline
\end{tabular}

${ }^{\dagger}$ EV disponibles en febrero: es la cantidad de EV totales correspondiente al balance forrajero evaluado a fines de febrero.

The Cow Equivalent (CV) available in February is the balance CV evaluated at the end of February.

‡ El porcentaje de recría es el número de animales engordados sobre el número total de animales destetados multiplicado por 100 .

The percentage of fattening is the number of finished animals over the total number of weaned animals times 100 . 
correspondiente a los períodos Futuro Cercano y Futuro Lejano. Este procedimiento asegura no introducir ningún sesgo por efecto precios en el cálculo del margen bruto, y asume que el cambio climático tendrá un efecto neutro en la estructura de precios de los animales vendidos. Supuestos similares han sido utilizados en estudios previos del impacto del cambio y la variabilidad climática (Letson and McCullough, 2001), además un estudio reciente concluye que la cadena de producción y procesamiento de carne vacuna del Noroeste de Estados Unidos experimentará solo cambios mínimos como consecuencia del cambio climático, en parte debido a las capacidades de regulación biológica del ganado bovino (Hristov et al., 2018). Para hacer comparable el valor de compra de las ventas a través de los años, los precios fueron indexados por medio del Índice de Precios Mayoristas (IPIM), (INDEC, 2017) y expresados en pesos constantes de marzo de 2017. Para los restantes componentes del costo se tomaron los precios vigentes a marzo de 2017 por medio de consultas con comercios especializados y consignatarios de la localidad de Balcarce. Para el precio del maíz utilizado como suplemento se tomó el precio promedio de este grano durante el período Histórico. Finalmente, se realizaron análisis de sensibilidad variando de uno en uno y en $10 \%, 15 \%$ y $20 \%$ en más y en menos los precios de los productos vendidos y de los dos principales componentes del costo, gastos de comercialización y fletes y alimentación.

Los cálculos de balance forrajero, producción de carne y margen bruto fueron realizados en planillas Excel ${ }^{\circledR}$. La significancia estadística de los cambios en las variables de clima, pasto, producción de carne y margen bruto se evaluaron mediante el test de Kruskal-Wallis y el test de Wilcoxon, dada la distribución no-normal de las variables.

\section{RESULTADOS Y DISCUSIÓN}

El Panel A de la Fig. 1 muestra el ciclo anual medio de la temperatura mínima y máxima diaria en el sitio seleccionado durante los períodos analizados simulado por el modelo RCA3. Se observa un incremento de las temperaturas desde el período Histórico hacia el Futuro Lejano de 2,6 y $1,9^{\circ} \mathrm{C}$ para la T-min y T-max, respectivamente. Los valores de T-min y T-max mostraron incrementos significativos $(\mathrm{p}<0,05)$ entre los tres períodos analizados en los 12 meses del año.

El Panel B de la Fig. 1 muestra el ciclo anual de la precipitación media para los 3 períodos analizados. Se evidencia un incremento en las precipitaciones en el Futuro Cercano y en el Futuro Lejano para todos los meses del año, con incrementos promedios de 25 y $271 \mathrm{~mm}$ año ${ }^{-1}$, respectivamente. Sin embargo, a diferencia de las temperaturas, las precipitaciones presentaron una gran variabilidad inter-anual (no mostrada en la figura) y solo se registraron incrementos estadísticamente significativos $(p<0,05)$ en 4 de los 12 meses del año.

La Tabla 2 muestra la intensidad de la señal de cambio climático evaluado a través de la relación incremento vs variabilidad promedio anual (cociente $R_{v a r}$ ). Esta señal muestra una intensidad alta para Tmin y Tmax con valores del indicador $R_{v a r}$ claramente superiores a 1. Sin embargo, la señal de cambio para las precipitaciones presenta una intensidad moderada, con valores de $R_{v a r}$ inferiores o cercanos a 1 . Estos resultados indican que la proyección de precipitación es menos confiable que la de temperatura. Resultados similares han sido hallados para el área bajo estudio por Cabré et al. (2016). Los datos presentados sugieren una tendencia clara de incremento de la temperatura de unos $2^{\circ} \mathrm{C}$ en promedio y un incremento moderado de las precipitaciones hasta finales del siglo.

La Fig. 2 muestra la producción de pasto mensual para cada uno de los períodos analizados. La producción promedio de pasto disminuye levemente en el Futuro Cercano, en comparación con el período Histórico, para volver a incrementarse en el Futuro Lejano. El ciclo anual de producción se mantiene, con un mayor crecimiento del pasto durante la primavera y el otoño y con producciones mínimas durante el invierno, coincidiendo con el aumento de temperatura y régimen de lluvias esperado en el futuro. Sin embargo, la producción

Tabla 2. Intensidad de la señal de cambio climático, $\mathbf{R}_{v a r^{\prime}}$ para T-min, $T$-max y precipitación para cada período analizado.

Table 2. Climate change signal-to-noise ratio intensity, $\mathbf{R}_{v a r^{\prime}}$ for T-min, T-max and precipitation in each period analyzed.

\begin{tabular}{lccc}
\hline & Histórico & Futuro Cercano & Futuro Lejano \\
\hline Histórico & - & 1,$92 ; 2,41 ; 0,18$ & 7,$28 ; 3,55 ; 1,13$ \\
Futuro Cercano & & - & 3,$90 ; 1,39 ; 1,24$ \\
Futuro Lejano & & & - \\
\hline
\end{tabular}




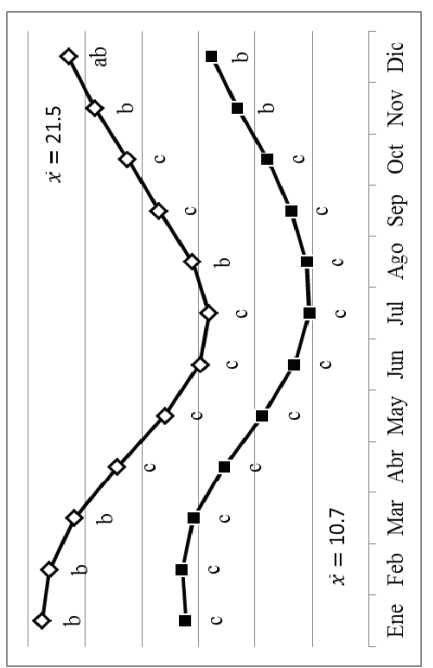

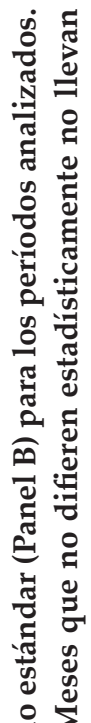
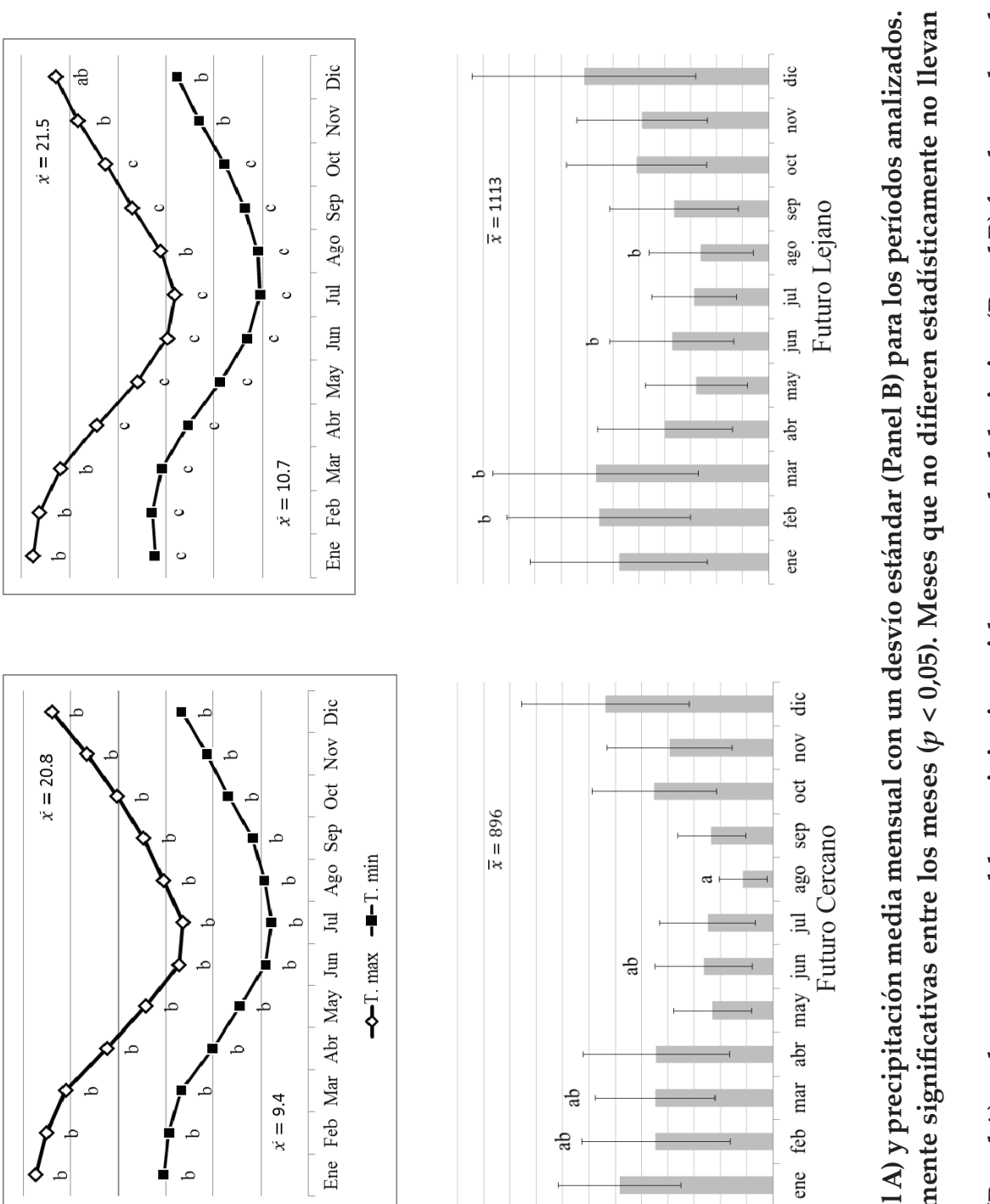

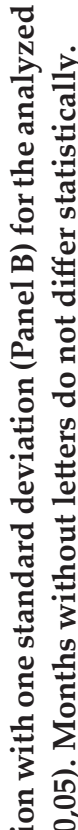

过

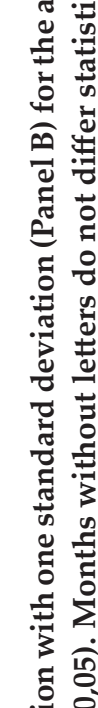

$\Xi \vee$

ป 2

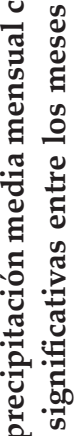

تृ

\&.

टे

롤

ฮิ

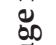

晃

ฮ

ฮี :

文

๙

文苋

幽

ฮ छ
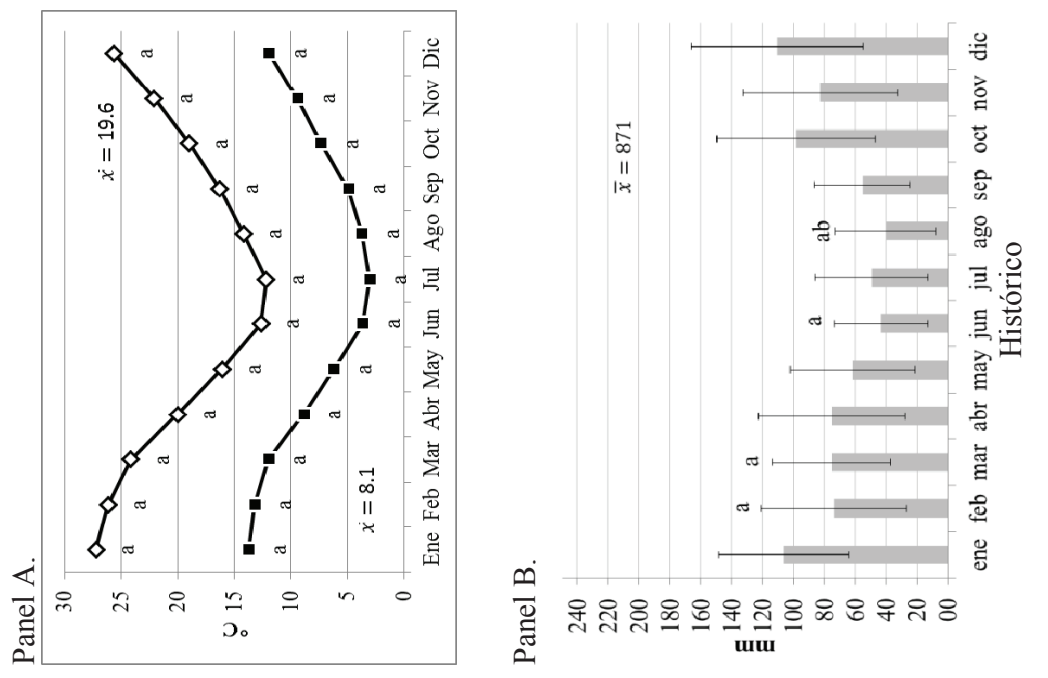

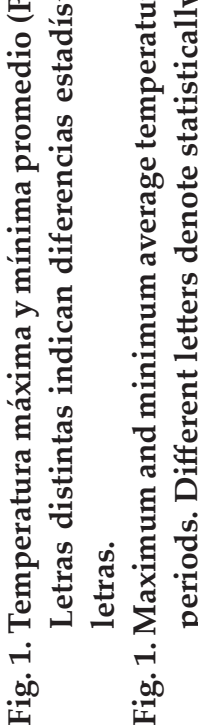

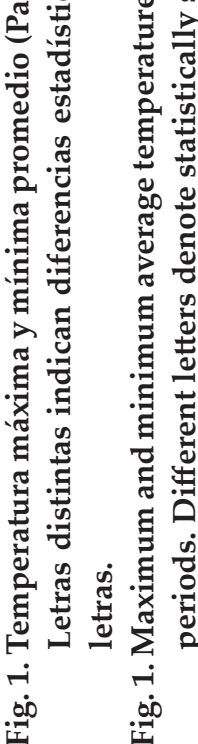




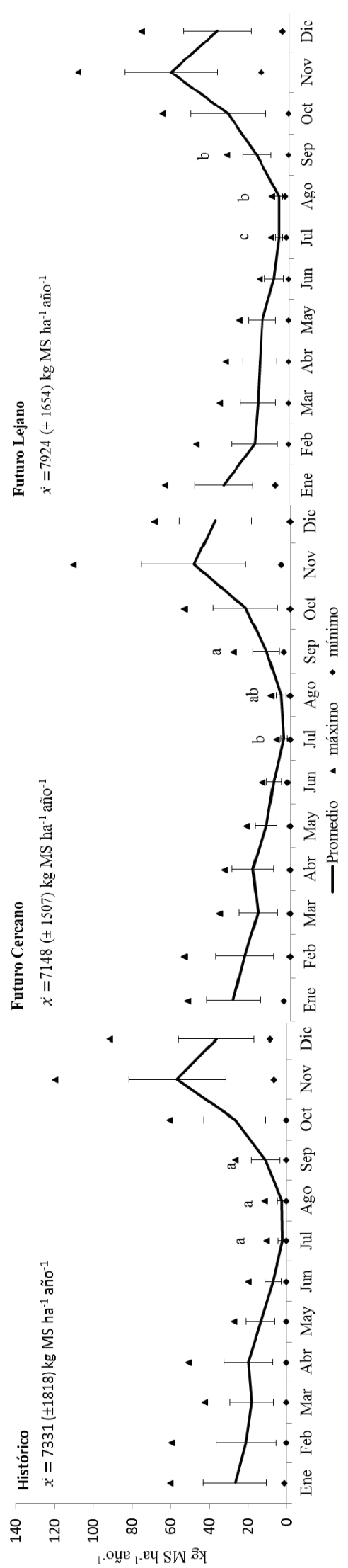

mensual presenta diferencias estadísticamente significativas $(p<0,05)$ entre períodos en solo 3 de los 12 meses del año - julio, agosto y septiembre. Dadas las mayores temperaturas y precipitaciones anuales en los períodos Futuro Cercano y Futuro Lejano la producción de pasto se incrementa significativamente en estos meses, pero la variabilidad interanual de las lluvias podría estar limitando un incremento más generalizado en la producción vegetal. Además, el incremento de las lluvias es en parte contrarrestado por la mayor evapotranspiración resultante de las mayores temperaturas (Thornton et al., 2009; Izaurralde et al., 2011). Como resultado, el incremento de producción de pasto es de escasa magnitud.

Al igual que la producción de pasto, la producción de carne promedio del período Histórico de $187 \mathrm{~kg} \mathrm{ha}^{-1}$, disminuye levemente en el Futuro Cercano hasta un promedio de $177 \mathrm{~kg} \mathrm{ha}^{-1}$, para volver a incrementarse en el Futuro Lejano, a un promedio de $188 \mathrm{~kg} \mathrm{ha}^{-1}$ (Fig. 3, Panel A). A su vez, la variabilidad de esta producción sigue un patrón similar, incrementándose levemente en el Futuro Cercano para luego disminuir en el Futuro Lejano. El panel B de la Fig. 3 muestra el margen bruto obtenido por el rodeo simulado durante los tres períodos analizados. El margen bruto muestra un patrón similar al de la producción de carne, disminuyendo levemente a $1697 \$$ ha $^{-1}$ (el tipo de cambio promedio durante marzo de 2017 fue de 1 US\$ = 15.52 \$) en el Futuro Cercano, para incrementarse a $1760 \$$ ha $^{-1}$ en el Futuro Lejano. Este margen bruto tiende a volverse más estable en los períodos Futuro Cercano y Futuro Lejano, comparado con el período Histórico. Dado que las diferencias en la producción de pasto no son de magnitud entre los períodos analizados, ni la producción de carne ni el margen bruto muestran diferencias significativas entre períodos

Los resultados presentados muestran que las condiciones para la producción de pasto tienden a mejorar levemente en los períodos futuros dadas las mayores temperaturas y los incrementos moderados en las precipitaciones promedio. Sin embargo, una mayor variabilidad de las precipitaciones en los períodos futuros limitarían incrementos mayores en la producción de pasto, carne y en el margen bruto logrado (Thornton et al., 2009; Henry et al., 2018). Estas condiciones hacen que en el Futuro Lejano podría mantenerse una mayor carga animal, engordando todos los animales producidos en un mayor número de años que en los otros períodos. Estas mejores condiciones de crecimiento vegetal se reflejan en producciones de carne y márgenes brutos levemente mayores y más estables en el Futuro Lejano. Es necesario considerar que este 
Panel A: Producción de Carne

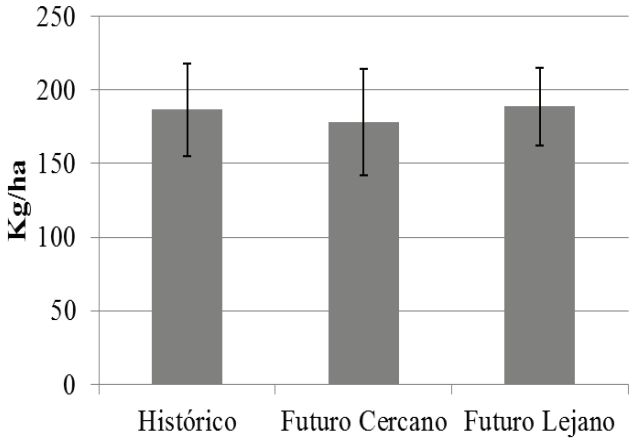

Panel B: Margen bruto

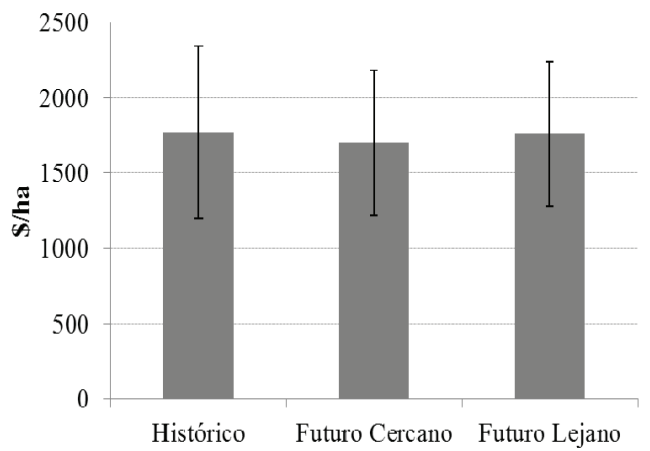

Fig. 3. Producción de carne y margen bruto para cada período analizado.

Fig. 3. Meat production and gross margin for each period analyzed.

trabajo no considera las contribuciones de la ganadería al cambio climático vía emisiones de gases de efecto invernadero. La cuantificación de estas contribuciones requeriría de estudios especializados que exceden el objetivo planteado aquí.

La Fig. 4 muestra la distribución de probabilidad acumulada del margen bruto ganadero en cada período. Las distribuciones de probabilidad del período Histórico y del Futuro Cercano alcanzan márgenes brutos similares en el rango de probabilidad del $10 \%$ al $80 \%$. La distribución del período Futuro Cercano tiene un mínimo menor que las correspondientes a los otros períodos. Esto indicaría que el período Futuro Cercano el margen bruto mínimo podría ser menor que en los otros períodos; sin embargo, las diferencias son muy pequeñas.

En cambio el Futuro Lejano se presenta como el período más favorable de los tres. La distribución de probabilidad de este período presenta mayores mínimos y se ubica a la derecha de las restantes en el rango de probabilidad de $0 \%$ a $50 \%$. Es decir, en el Futuro Lejano existe un $50 \%$ de probabilidad de obtener márgenes brutos levemente mayores que en los otros períodos. Para probabilidades mayores al $50 \%$, las tres distribuciones son muy similares entre sí, excepto que las distribuciones correspondientes al Futuro Cercano y Futuro Lejano presentan máximos levemente inferiores al período Histórico. A pesar de estas diferencias, las tres funciones de distribución acumulada del margen bruto no difieren estadísticamente entre sí según el test de Kolmogorov-Smirnov.

\section{CONCLUSION}

Este trabajo ha estimado el impacto del cambio climático en la productividad de un rebaño de producción ganadera de ciclo completo del sudeste bonaerense. Los resultados muestran una tendencia clara de aumento de temperatura de unos $2^{\circ} \mathrm{C}$ hacia finales del siglo, y una tendencia más variable de incremento de precipitaciones, en unos $217 \mathrm{~mm}$ año ${ }^{-1}$, en promedio, entre el período Histórico y el Futuro Lejano. Estos patrones climáticos causan una disminución leve en el crecimiento del pasto en el Futuro Cercano y un incremento moderado en la producción de pasto en el Futuro Lejano, con respecto al período Histórico. A través de los períodos analizados, el ciclo anual de crecimiento del forraje se mantiene.

Consistente con las variaciones en producción de pasto, la producción de carne y el margen bruto presentan descensos leves en el Futuro Cercano e incrementos moderados en el Futuro Lejano, en promedio. El Futuro Lejano presenta las mejores condiciones para el crecimiento del forraje, soportando la carga animal más alta y permitiendo obtener la mayor producción de carne y el mayor margen bruto de los tres períodos. Las distribuciones de probabilidad del margen bruto son similares para los tres períodos, indicando que el riesgo económico de la producción ganadera se mantendría, o incluso disminuiría levemente en el Futuro Lejano, comparado con los niveles actuales.

Este trabajo constituye uno de las primeras evaluaciones del impacto del cambio climático sobre la productividad ganadera en la región pampeana y contribuye a caracterizar los niveles medios y la variabilidad esperada en productividad, tanto en términos físicos como monetarios. 


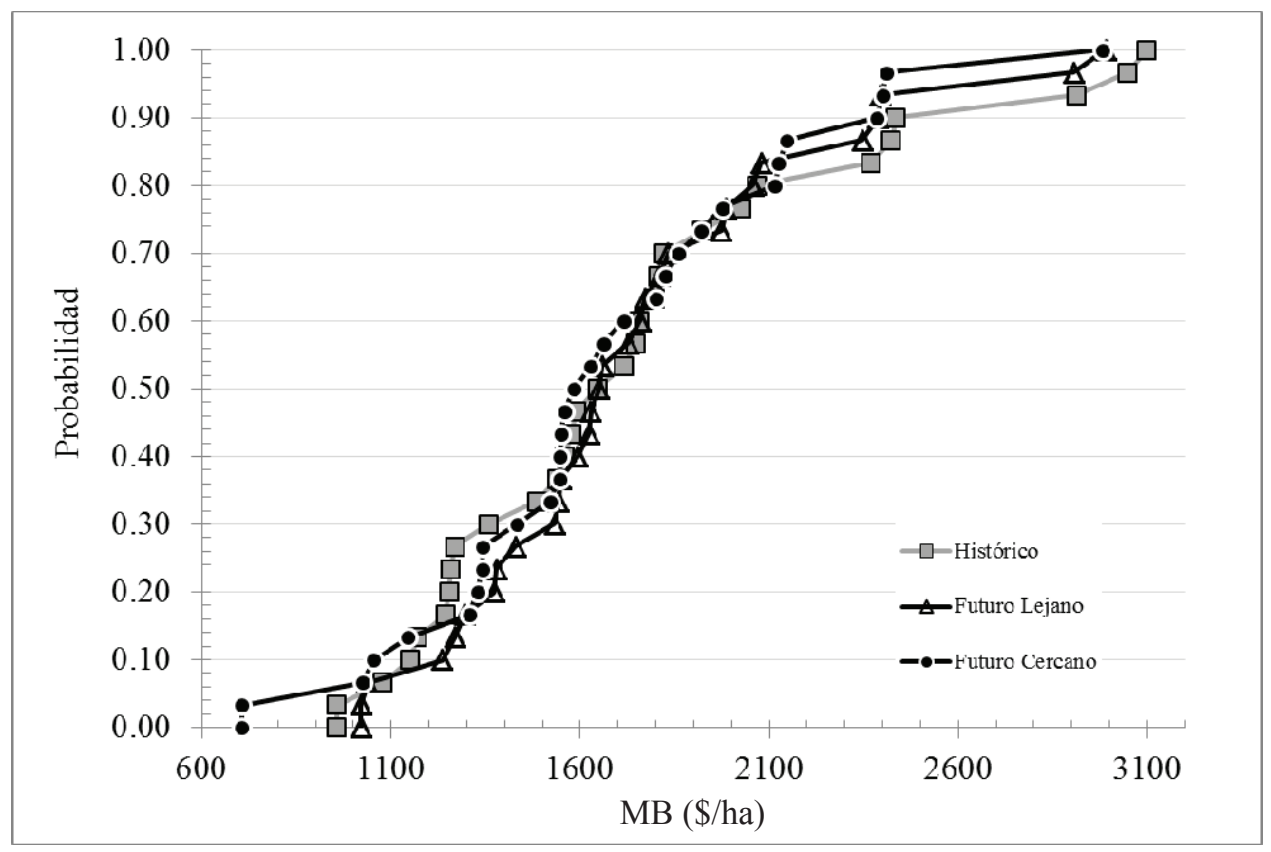

Fig. 4. Distribución acumulada del margen bruto para los períodos analizados.

Fig. 4. Cumulative distribution functions for the gross margin for each period analyzed.

\section{AGRADECIMIENTOS}

Este trabajo ha recibido financiamiento de los siguiente Proyectos de INTA, PNPA 1126022 y PNSEPT 1129023. Este trabajo incluye análisis iniciales del trabajo de beca del estudiante Julian Peñalva, a quien los autores agradecen su colaboración.

\section{LITERATURA CITADA}

Alexandris, S., R. Stricevic, and S. Petkovic. 2008. Comparative analysis of reference evapotranspiration from the surface of rainfed grass in central Serbia, calculated by six empirical methods against the PenmanMonteith formula. Eur. Water 21(22):17-18.

ARC. 1980. The nutrient requirements of ruminant livestock. 4th ed. Agricultural Research Council (ARC). CAB International, Wallingford, United Kingdom.

Burges, J.C., and A.J. Romera. 2007. Estimación de la variabilidad del crecimiento de las pasturas para presupuestos forrajeros. Rev. Arg. Prod. Anim. 27(1):127-128.
Burges, J.C., Á. Romera, F.G. Continanza, C. Faverín, A. Sciotti, and H.H. Fernández. 2014. Reglas de manejo para sistemas pastoriles de cría vacuna. Evaluación del sistema "SIMPLE." p. 189-190. En Burges, J.C., Faverín, C., Aello, M.S., Recavarren, P. (eds.). Experiencias prácticas sobre ganadería en el territorio Centro Sur de Buenos Aires. Ediciones INTA, Buenos Aires, Argentina.

Cabré, M.F., S. Solman, and M. Núñez. 2016. Regional climate change scenarios over southern South America for future climate (2080-2099) using the MM5 Model. Mean, interannual variability and uncertainties. Atmósfera 29(1):35-60.

Christensen, J.H., K. Krishna Kumar, E. Aldrian, S.-I. An, I.F.. Cavalcanti, M. de Castro, et al. 2013. Climate phenomena and their relevance for future regional climate change. p. 1217-1308. In Stocker, T.F., Qin, D., Plattner, G. -K., Tignor, M., Allen, S.K., Boschung, J., Nauels, A., Xia, Y., Bex, V., Midgley, P.M. (eds.), Climate Change 2013 the Physical Science Basis: Working Group I Contribution to the Fifth Assessment Report of the Intergovernmental Panel on Climate Change. Cambridge University Press, Cambridge, United Kingdom. 
CLARIS-LPB. 2010. Recommendations on methods and datasets for regional climate projections. Facultad de Ciencias Exactas y Naturales, Universidad de Buenos Aires, Argentina. Reporte de avance: Deliverable 7.3. Disponible en http://eolo.cima.fcen.uba. ar/LPB/lpb_listaDeliverables_products.php (Consulta 11 abril 2018).

Giménez, A. 2006. Climate change and variability in the mixed crop/livestock production systems of the Argentinean, Brazilian and Uruguayan Pampas. Final Report AIACC Project No. LA 27. International START Secretariat, Washington, DC, USA.

Golluscio, R. 2009. Receptividad ganadera: marco teórico y aplicaciones prácticas. Ecol. Austral 19:215-232.

Hayashi, Y. 1982. Confidence intervals of a climatic signal. J. Atmos. Sci. 39(9):1895-1905. doi: 10.1175/1520-0469(1982)039<1895:CIOA CS>2.0.CO;2.

Hay, L.E., R.L. Wilby, and G.H. Leavesley. 2000. A comparison of delta change and downscaled GCM scenarios for three mountainous basins in the United States. J. Am. Water Resour. Assoc. 36(2):387-397. doi: 10.1111/j.17521688.2000.tb04276.x.

Henry, B.K., R.J. Eckard, and K.A. Beauchemin. 2018. Review: Adaptation of ruminant livestock production systems to climate changes. Animal: 1-12. doi: 10.1017/ S1751731118001301.

Hristov, A.N., A.T. Degaetano, C.A. Rotz, E. Hoberg, R.H. Skinner, T. Felix, et al. 2018. Climate change effects on livestock in the Northeast US and strategies for adaptation. Clim. Change 146(1-2):33-45. doi: 10.1007/ s10584-017-2023-z.

INDEC. 2017. Instituto Nacional de Estadísticas y Censos (INDEC), República Argentina. Disponible en https://www.indec.gob.ar/ (Consulta 8 octubre 2017).

Izaurralde, R.C., A.M. Thomson, J.A. Morgan, P.A. Fay, H.W. Polley, and J.L. Hatfield. 2011. Climate impacts on agriculture: Implications for forage and rangeland production. Agron. J. 103(2):371-381. doi: 10.2134/ agronj2010.0304.

Jones, C., U. Willén, A. Ullerstig, and U. Hansson. 2004. The Rossby Centre Regional Atmospheric Climate Model. Part I: Model Climatology and Performance for the Present Climate over Europe. Ambio 33(June):199210.
Keaokliang, O., T. Kawashima, W. Angthong, T. Suzuki, and R. Narmseelee. 2018. Chemical composition and nutritive values of cassava pulp for cattle. Anim. Sci. J. 89(8):1120-1128. doi: 10.1111/asj.13039.

Letson, D., and B.D. McCullough. 2001. ENSO and soybean prices: Correlation without causality. J. Agric. Appl. Econ. 33(3):513-522.

Magrin, G.O., M.I. Travasso, and G.R. Rodríguez. 2005. Changes in climate and crop production during the 20th century in Argentina. Clim. Change 72(1-2):229-249.

Marengo, J.A., T. Ambrizzi, R.P. da Rocha, L.M. Alves, S. V. Cuadra, M.C. Valverde, et al. 2009. Future change of climate in South America in the late twenty-first century: intercomparison of scenarios from three regional climate models. Clim. Dynam. doi: 10.1007/s00382-009-0721-6.

Michelangeli, P.-A., M. Vrac, and H. Loukos. 2009. Probabilistic downscaling approaches: Application to wind cumulative distribution functions. Geophys. Res. Lett. 36:1-14.

Oettli, P., B. Sultan, C. Baron, and M. Vrac. 2011. Are regional climate models relevant for crop yield prediction in West Africa? Environ. Res. Lett. 6(1):40-48.

Priestley, C.H.B., and R.J. Taylor. 1972. On the Assessment of Surface Heat Flux and Evaporation Using Large-Scale Parameters. Mon. Weather Rev. 100(2):81-92. doi:0.1175/1520-0493(1972)100<0081:OTAOS $\mathrm{H}>2.3 . \mathrm{CO} ; 2$.

Quiroz, J.L., and A. Rodríguez. 2012. Calendario sanitario para la ganadería de cría. Kit de extensión para las pampas y campos. Fundación Vida Silvestre, Buenos Aires, Argentina.

Re, M., and V.R. Barros. 2009. Extreme rainfalls in SE South America. Clim. Change 96(1):119136. doi: 10.1007/s10584-009-9619-x.

Romera, A. 2004. Modelling of management strategies for pastoral cow-calf farms in the Salado Region of Argentina. Ph.D. Thesis. Animal Science Dept., Massey University. Available at https://muir.massey.ac.nz/ bitstream/10179/1736/1/02_whole.pdf (Accessed 11 april 2018).

Romera, A.J., J.C. Burges, S.T. Morris, J. Hodgson, and S.J.R. Woodward. 2008. Modelling spring and autumn calving systems in beef herds of the Salado region of Argentina. Livest. Sci. 115(1):62-72. doi: 10.1016/j.livsci.2007.06.013.

Romera, A.J., D.G. McCall, J.M. Lee, and M.G. Agnusdei. 2009. Improving the McCall herbage growth model. New Zeal. J. Agric. Res. 52(4):477-494. doi: 10.1080/00288230909510529. 
Romera, A.J., S.T. Morris, J. Hodgson, W.D. Stirling, and S.J.R. Woodward. 2004. A model for simulating rule-based management of cow-calf systems. Comput. Electron. Agric. 42(2):67-86. doi: 10.1016/S01681699(03)00118-2.

Romera, A.J., S.T. Morris, J. Hodgson, W.D. Stirling, and S.J.R. Woodward. 2005a. Comparison of haymaking strategies for cow-calf in the Salado Region of Argentina using a simulation model. 3. Exploratory risk assessment. Grass Forage Sci. 60:417-422.

Romera, A.J., S.T. Morris, J. Hodgson, W.D. Stirling, and S.J.R. Woodward. 2005b. Comparison of haymaking strategies for cow-calf in the Salado Region of Argentina using a simulation model. 2. Incorporation of flexibility in the decision rules systems. Grass Forage Sci. 60(September):409-416.

Samuelsson, P., C.G. Jones, U. Willén, A. Ullerstig, S. Gollvik, U. Hansson, et al. 2011. The Rossby Centre Regional Climate model RCA3: model description and performance. Tellus A 63(1):4-23. doi: 10.1111/j.16000870.2010.00478.x.

Sánchez, E., S. Solman, A.R.C. Remedio, H. Berbery, P. Samuelsson, R.P. Da Rocha, et al. 2015. Regional climate modelling in CLARIS-LPB: a concerted approach towards twenty first century projections of regional temperature and precipitation over South America. Clim. Dynam. 45(7-8):2193-2212. doi: 10.1007/ s00382-014-2466-0.

Seo, S.N. 2009. A micro-econometric analysis of the impact of climate change on livestock management in South America. Quarterly, J. Int. Agric. 48(3):265-282.

Seo, S.N., B.A. McCarl, and R. Mendensohn. 2010. From beef cattle to sheep under global warming? An analysis of adaptation by livestock species choice in South America. Ecol. Econ. 69(12):2486-2494.
Skansi, M. de los M., M. Brunet, J. Sigró, E. Aguilar, J.A. Arevalo Groening, O.J. Bentancur, et al. 2013. Warming and wetting signals emerging from analysis of changes in climate extreme indices over South America. Global Planet. Change 100:295-307. doi: 10.1016/j. gloplacha.2012.11.004.

Solman, S.A., E. Sanchez, P. Samuelsson, R.P. Rocha, L. Li, J. Marengo, et al. 2013. Evaluation of an ensemble of regional climate model simulations over South America driven by the ERA-Interim reanalysis: model performance and uncertainties. Clim. Dynam. 41(5):1139_ 1157. doi: 10.1007/s00382-013-1667-2.

Thornton, P.K., J. van de Steeg, A. Notenbaert, and M. Herrero. 2009. The impacts of climate change on livestock and livestock systems in developing countries: A review of what we know and what we need to know. Agric. Syst. 101(3):113-127. doi: 10.1016/j. agsy.2009.05.002.

Tosi, J.C. 2017. Ganadería en sistemas agrícolas: precios ganaderos y cambio tecnológico en ciclo completo. INTA. Reporte online de Junio. 5 p. Disponible en https://inta.gob.ar/ documentos/ganaderia-en-sistemas-agricolas-precios-ganaderos-y-cambio-tecnologico-en-ciclo-completo-0 (Consulta 4 octubre 2017).

Yang, W., J. Andréasson, L. Phil Graham, J. Olsson, J. Rosberg, and F. Wetterhall. 2010. Distribution-based scaling to improve usability of regional climate model projections for hydrological climate change impacts studies. Hydrol. Res. 41(3-4):211-229. doi: 10.2166/ nh.2010.004. 